\title{
The Evolution of Information Resource Allocation Research based on Knowledge Mapping
}

\author{
Qiu Junping ${ }^{1}$ and Lou Wen ${ }^{2}$ \\ ${ }^{1,2}$ Research center for Chinese science evaluation, Wuhan University \\ jpqiu@whu.edu.cn, ${ }^{2}$ hotwen_l@sina.com
}

\begin{abstract}
Based on the bibliography references included in ISI Web of Knowledge, and by use of visualization network analysis tool_Citespace to conduct co-citation and cluster analysis of collected data, this paper renders knowledge mapping on the development and evolution of information resource allocation research, reveals two different periods of information resource allocation research, and identifies representative scientists as well as relevant literatures in each period. Meanwhile, this paper demonstrates the hot research fields represented by enterprise resource allocation, theory and practice research of information resource allocation, biological information resource allocation, retrieval and sharing research, and computer network communication resource allocation, and further presents the frontiers represented by empirical research of information resource allocation, information retrieval about resource allocation, network communication resource allocation, biological information data mining, biology knowledge management, gene-ontology and semantic web.
\end{abstract}

Keywords: information resource allocation; knowledge mapping; Citespace; research evolution

\section{Introduction}

Information resource allocation is one of the most important research areas of information resource management and information economics. Researchers of information resource allocation aim to realize equitable allocation and maximum utility of information resource. Information resource allocation involves in universities, governments, enterprises and other allocation subjects, as well as information resource users, who are straight beneficiary of information resource effectiveness [1, 2]. However, with the development of information technology, human beings need to cope with more and more problems of the process of information resource allocation. This circumstance catches scholars and industries attention. Experts of information resource allocation research have published many achievements, such as research papers, books, and reviews. As we can see, research papers did discover models and methods to optimize information resource allocation, and reviews showed details of information resource allocation research in the world or used bibliometrics to summarize current situation of this area. Indeed, these articles provided state of development of information resource allocation research [3-5]. However, they do not present history, development, hot topics and front trends of information resource allocation research systematically, especially by using the most impressive way [6-8].

This article aims at visualizing and quantitative analysis of the history, development, hotspots and trends of information resource allocation research. We use Citespace, a dynamic visualization analysis tool, to draw a timeline map of information resource allocation research evolution [9]. According to the map, we divide information resource allocation research into 
several periods, and describe representatives and their contribution of identical periods. Meanwhile, using Citespace function and burst term detective algorithm, we find out hot topics and front trends of information resource allocation research, so that other researchers can understand the whole picture of information resource allocation research.

\section{Methodology}

Data source of this paper comes from SCIE, SSCI and A\&HCI. Search query is "TS=information resource allocat* OR TS=information resource optimiz* OR TS=information resource distribut*". Time period is chosen "all". The date of data collection is July 27th in 2012. The resultant dataset contains a total of 6,466 records. A bibliographic record in database contains fields such as author, title, abstract, and references.

This paper uses the information visualization software, Citespace, which is developed by Chaomei Chen. With the help of Citespace, we draw the timeline map of information resource allocation representatives, the knowledge mapping of hot topics, and the knowledge mapping of front trends in order to directly describe theoretical source and research context.

\section{Knowledge Mapping of Development of Information Resource Allocation Research}

We import 6,466 records to Citespace, and select proper threshold $(3,3,17 ; 4,4,20 ; 5,5$, 23 ) to get the timeline map (Figure 1). In timeline map, a node represents a paper. The size of a node represents citation frequency. The relations between papers are represented by lines between nodes. The thickness of lines implies co-citation frequency of papers. The color of each circle of nodes represents the citation year. In Figure 1, there are 366 nodes and 2,312 lines.

The very first cited paper of information resource allocation research appears in 1935, which implies that as one of the most important areas of information management research, information resource allocation research has a history of nearly eighty years. According to the distribution of citation time and citation frequency in timeline map, we divided the evolution of information resource allocation research into two periods. Foundation period is from 1935 to 1989 , and developing period is from 1990 to nowadays.

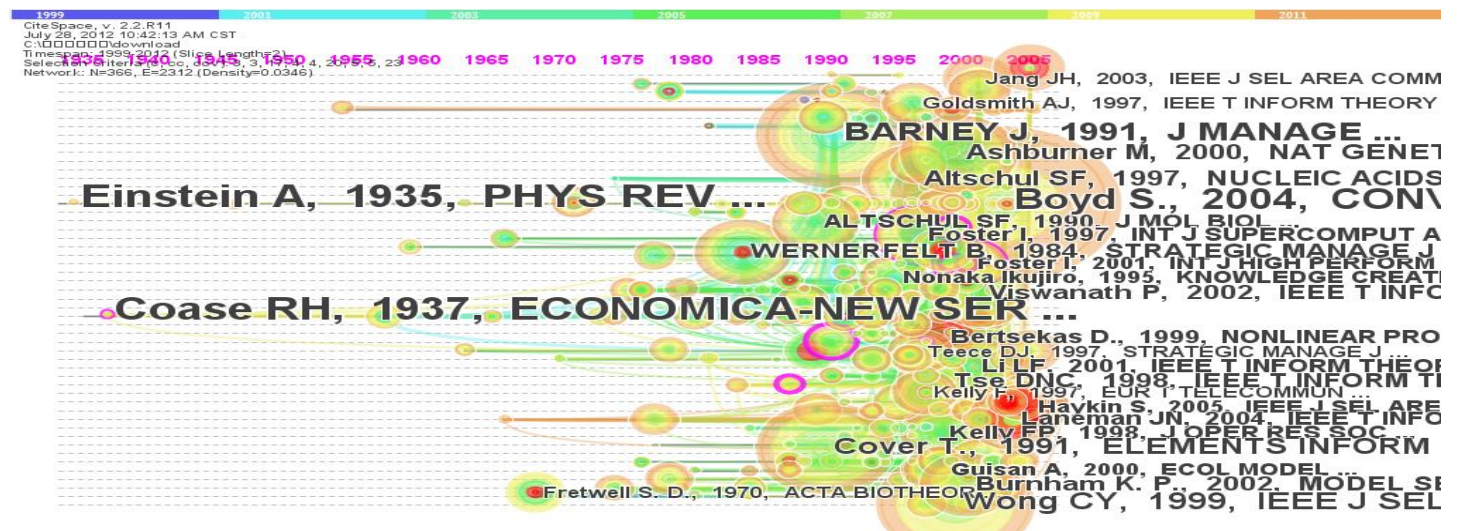

Figure 1. Timeline Map of Representatives and Works of Information Resource Allocation Research. We Choose Einstein and Coase to be Specially Marked because they are Two of Ancestors In Timeline Map 


\subsection{Foundation Period (1935-1989)}

The very first paper is "Can Quantum-Mechanical Description of Physical Reality Be Considered Complete?" which was written by Einstein [10], and published at Physical Review. Einstein argued that every conclusion cannot be made unless after being measured or practiced. His paper focuses on the importance of experiments, which has an effect on empirical study of information resource allocation.

The other early paper is written by Coase [11]. His paper was one of the first to use transaction cost to analyze the relation between firm and market. Coase noted that the nature of the firm was resource allocation mechanism, so was the nature of the market. The difference between firm and market is that resource was allocated by enterprise management organization in a firm, while resource was allocated by price mechanism in the market. Managers should make allocation decisions by digging out the relation between enterprise organization cost and market price. Coase's paper has affected enterprise resource allocation research in the long run.

Penrose published "The Theory of the Growth of the Firm" [12], explored factors and mechanism of the growth of the firm, and designed resource-competitiveness-growth model. She suggested that firm resource brought about competitiveness, competitiveness had an effect on requirements to encourage production of the firm, so firm got developed. Thus, resource is the source of market competitiveness and innovation. Organization structure, management and knowledge creation of the firm cannot leave without resource.

Fretwell attempted to analyze the affection on natural resource allocation mechanism and model of social environment, biological behavioral and mentality [13]. In Figure 1, the node of Fretwell is one of burst nodes, which means Fretwell's paper catches scholars' attention suddenly in the 2010s, and led bioinformatics resource allocation to one of resource allocation areas.

Following Coase and Penrose's research, Wernerfelt published "A resource-based view of the firm" to propose resource-based theory of the firm [14]. The resource-based theory agreed with Penrose, and demonstrated that a firm should focus on resource rather than product. Wernerfelt also designed a model based on resource position and resource-product. He suggested that optimal competitive strategies were based on these resources, and described how current resources could be used to develop new ones. We can see that in Figure 1 the node of Wernerfelt is the biggest one, so the idea of Wernerfelt was one of the most effective papers in the 1980s.

In the foundation period, we can see some nodes which are not so much shining but important (Details in Table 1). They are closely related to information theory and mathematics. For example, the classic work, which was written by Shannon [15], "A Mathematical Theory of Communication", constructed mathematics model of information system, and proposed information source, information channel and other important results. Another highly cited paper is written by Miller [16]. He tested a large-scale dataset to show that if unaided observers took advantages of information techniques, they would break information bottleneck. Shannon, Miller and other representatives' contribution have made a great success on information theory and communication resource allocation. 
Table 1. Highly Cited Representatives in Foundation Period (citation frequency>6). We Choose these Representatives to Show in Table 1, because Citation Frequency of No.1 and 2 are Bigger than 31 In Timeline Map, and Citation Frequency of No. 3 to 7 are Bigger than 6 before 1960 (the early Foundation Period)

\begin{tabular}{lllll}
\hline$\#$ & Author & Year & Representative source & Frequency \\
\hline 1 & WERNERFELT B & 1984 & STRATEGIC MANAGE J & 48 \\
2 & Fretwell S. D. & 1970 & ACTA BIOTHEOR & 33 \\
3 & Penrose E & 1959 & THEORY GROWTH FIRM & 20 \\
4 & MILLER GA & 1956 & PSYCHOL REV & 16 \\
5 & Coase RH & 1937 & ECONOMICA-NEW SER & 10 \\
6 & Bellman R. & 1957 & DYNAMIC PROGRAMMING & 10 \\
7 & SHANNON CE & 1948 & AT\&T TECH J & 9 \\
8 & Zipf George Kingsley & 1949 & HUMAN BEHAV PRINCIPL & 9 \\
9 & HUTCHINSON GE & 1957 & COLD SPRING HARB SYM & 9 \\
10 & Hayek FA & 1945 & AM ECON REV & 8 \\
11 & Einstein A & 1935 & PHYS REV & 7 \\
\hline
\end{tabular}

\subsection{Developing Period (1935-1989)}

Since 1990, the nodes in timeline map became intensive, including some highly cited nodes. More and more famous works (Details in Table 2) told that information resource allocation research entered into a developing period. Scholars did deeper study to continue former researches. The main content of achievements includes four aspects.

Table 2. Highly Cited Representatives in Developing Period (Citation Frequency $>31$ )

\begin{tabular}{llll}
\hline Author & Year & Representative source & Frequency \\
\hline Boyd S. & 2004 & CONVEX OPTIMIZATION & 100 \\
BARNEY J & 1991 & J MANAGE & 73 \\
Wong CY & 1999 & IEEE J SEL AREA COMM & 72 \\
Cover T. & 1991 & ELEMENTS INFORM THEO & 61 \\
Ashburner M & 2000 & NAT GENET & 54 \\
Altschul SF & 1997 & NUCLEIC ACIDS RES & 50 \\
Viswanath P & 2002 & IEEE T INFORM THEORY & 47 \\
Tse DNC & 1998 & IEEE T INFORM THEORY & 46 \\
Burnham K. P. & 2002 & MODEL SELECTION MULT & 45 \\
Foster I & 1997 & INT J SUPERCOMPUT AP & 44 \\
ALTSCHUL SF & 1990 & J MOL BIOL & 43 \\
Kelly FP & 1998 & J OPER RES SOC & 42 \\
Laneman JN & 2004 & IEEE T INFORM THEORY & 42 \\
Bertsekas D. & 1999 & NONLINEAR PROGRAMMIN & 40 \\
Li LF & 2001 & IEEE T INFORM THEORY & 40 \\
Foster I & 2001 & INT J HIGH PERFORM C & 38 \\
Haykin S & 2005 & IEEE J SEL AREA COMM & 37 \\
Nonaka Ikujiro & 1995 & KNOWLEDGE CREATING C & 36 \\
Guisan A & 2000 & ECOL MODEL & 36 \\
Teece DJ & 1997 & STRATEGIC MANAGE J & 34 \\
Kelly F & 1997 & EUR T TELECOMMUN & 33 \\
Goldsmith AJ & 1997 & IEEE T INFORM THEORY & 32 \\
Jang JH & 2003 & IEEE J SEL AREA COMM & 32 \\
\hline
\end{tabular}

3.2.1. Enterprise Resource Allocation Research: In this period, as one of hot topics in foundation period, enterprise resource allocation research is still the core of information 
resource allocation research. The distribution of most of these nodes is in the early developing period. The node of Barney [17], who wrote "Firm Resources and Sustained Competitive Advantage", is the second biggest node in the whole map. Barney discussed four empirical indicators -- valuable, scarcity, imitability, and substitutability-- for measuring sustained competitive advantage of enterprise resource quality. His paper emphasized that the key to sustained competitive advantage of enterprise was that leader realized the importance of resource management. On the other hand, Nonaka Ikujiro's famous book, "The knowledge creating company" [18], described how Japanese understood and utilized knowledge on enterprise management. And Teece proposed dynamic capabilities and strategy management to catch up on the limitation of resource-based theory [19]. Teece divided enterprise resource into public resource, proprietary resources, organization ability, and innovation in order to deeply prove his dynamic capabilities framework. In all, these three academics discussed how enterprises sustained competitiveness from resource, knowledge and competitiveness aspects. Their studies prove that enterprise resource management research is one of branches of information resource management.

3.2.2. Theory and Methodology of Information Resource Allocation Research: As a classic work of information theory, "Elements of Information Theory" was one of the first to separate information resource allocation research from welfare economics research [20]. Since then, information resource allocation research became one of branches of information economics. Cover made every element and concept of information theory clear, and also explained how to use element of information theory to cope with problems, such as data compression, channel capacity, and network information flow. Burnham presented a general strategy for modeling and data analysis by using information theory methods [21]. Two of above carried forward Shannon's study to support theoretical research of information resource allocation.

Additionally, there are some nodes which suggest researches on information resource allocation methods. One of them is "Convex optimization", treated as the biggest node in the whole map [22]. They noted that convex optimization is a special nonlinear programming, which could be used for global optimization. And convex optimization was concluded that since it was good at resource integration, it has been applied to many areas, such as communication networks, signal processing, automatic control system, and data analysis. Meanwhile, Bertsekas wrote a book, named nonlinear programming, to side with large-scale resource allocation methods [23].

3.2.3. Allocation, Retrieval and Sharing of Bioinformatics Resource: After Fretwell, some scholars explored bioinformatics resource allocation research. Altschul invented a search tool to apply for biology science, which is BLAST (basic local alignment search tool) [24, 25]. BLAST is widely used for searching protein and DNA databases for sequence similarities. Also, Ashburner team created gene ontology to realize knowledge sharing of gene and protein [26]. Wildlife habitat was fully discussed and applied for forecasting habitat distribution [27].

3.2.4. Computer Network and Telecommunication Resource Allocation: Computer network and telecommunication resource allocation research was being a hot topic in developing period. In timeline map, ten of twenty-seven nodes represent computer network and telecommunication resource allocation research. Although these nodes are not bigger than some classic nodes, some of them are burst nodes as to be highly emphasis.

First of all, Foster and Kelly appear twice in Figure 1. Foster and Kesselman are pioneers of grid computing research. They did a series study to allocate distributed computer resource 
by using high speed network, and created Globus as grid computing standard to realize largescale communication and identity authentication [28, 29]. Meanwhile, Kelly's team had achievements on network congestion control and network transmission speed optimization. They developed NUM (Network Utility Maximization) framework to be widely applied for transmission speed allocation algorithm, Internet congestion control protocol and user behavior model [30].

There are many other academics studying communication resource allocation at the same time. For example, Goldsmith found capacity of fading channels with channel side information [31]. Throughput and delay-limited capacity region were characterized for multiaccess fading channels [32, 33]. Multiuser orthogonal frequency division multiplexing (OFDM) subcarrier, bit, and power allocation algorithm was proposed to minimize the total transmit power [34]. Wireless network resource research began to be discussed in the early 21 st century [35-37]. Since nodes of wireless network research are all burst nodes in timeline map, we assume that wireless network resource allocation research is creative as a new hot topic.

In all, information resource allocation originates from enterprise tradition resource allocation, develops to enterprise knowledge resource allocation and natural and biological resource allocation, and gets top with computer network and telecommunication resource allocation. All these areas refer to the whole information system, thus, information resource allocation research is going to be wide and deep, but not to the end.

\section{Knowledge Mapping of Hotspots and Front Trends of Information Resource Allocation Research}

Keywords indicate the core content of a paper. Analyzing highly citation frequency keywords can discover hot topics of information resource allocation research. Citespace also provides noun phrase detective algorithm to help for determining hot topics.

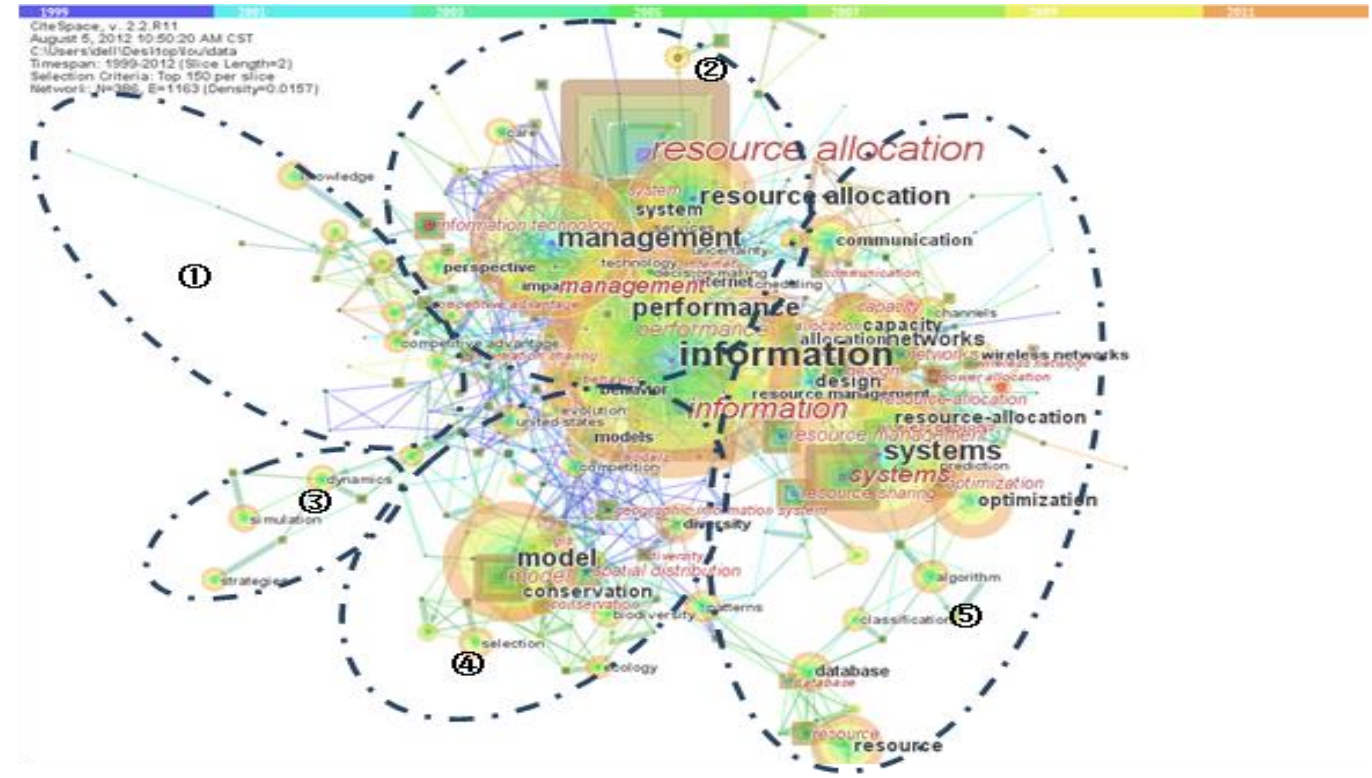

Figure 2. Knowledge Map of Hotspots of Information Resource Allocation Research. Circle with Dotted Line are Drew by Authors in Order to Make Five Classes Clear 


\subsection{Hotspots}

Also we import data into Citespace, and select keywords and noun phrase as network nodes and proper threshold (top 150 per slice). Figure 2 shows hotspots knowledge mapping of information resource allocation research. Square nodes represent noun phrase, while circle nodes represent keywords. The size of nodes represents their frequency of keywords and noun phrase. The bigger the node is, the hotter the topic is. And different color of nodes mean different citation year. In Figure 2, keywords and noun phrase are automatically clustered to five classes, which are coincidence with four subjects above.

The first class contains several keywords, such as knowledge, competitive advantages, knowledge sharing, and prospect. The thing that these keywords indicate is the same as the first content in developing period indicates, for example, the meaning of competitive advantages is the same as resource basement of enterprise resource allocation, the meaning of knowledge sharing is the same as knowledge that enterprise designs. So, enterprise resource allocation is the hop topic at that time.

The second and third class contains those keywords which are highly citation frequency keywords and noun phrase about theory and methodology, such as resource allocation, information, system, management, performance, and information technology, uncertainty in the second class, and dynamics, strategies in the third class. These keywords and noun phrase prove that theory of information resource and system dynamics are hot topics. Additionally, the second class has the highest frequency and these keywords and noun phrase have been cited at the whole years, which means theory and methodology of resource allocation has always been hot topics in information resource allocation research.

As for the fourth class, it contains model, conservation, diversity, competition, patterns, and ecology. These keywords are related to new technology of biological gene sequencing, dynamic gene ontology, biological habitat choice, geographical space distribution, distribution model and other bioinformatics resource allocation. And the fifth class is clearly related to computer network and telecommunication resource allocation, since the fifth class contains systems, design, database, optimization, networks, capacity, communication, and wireless networks. These keywords also have highly citation frequency, just lower than the second class, which means the developing telecommunication technology is a hot topic of resource allocation.

In addition, the lines which connect the second, third and fourth class are mainly purple. That means co-citation of the second, third and fourth class concentrates in the late 1990s. While, the lines which connect the second and fifth, first and second class are mainly green, which means their co-citation appears in the early 21 st century. Keywords co-citation shows crossed research hot topics. For instance, information, which is in the second class, is co-cited with model, which is in the fourth class in 1999. Then research about information model was a hotspot at that time. At a glance of Figure 1, some lines are yellow and orange. We find that those words' combination is hotspots nowadays, such as communication and resource allocation. 


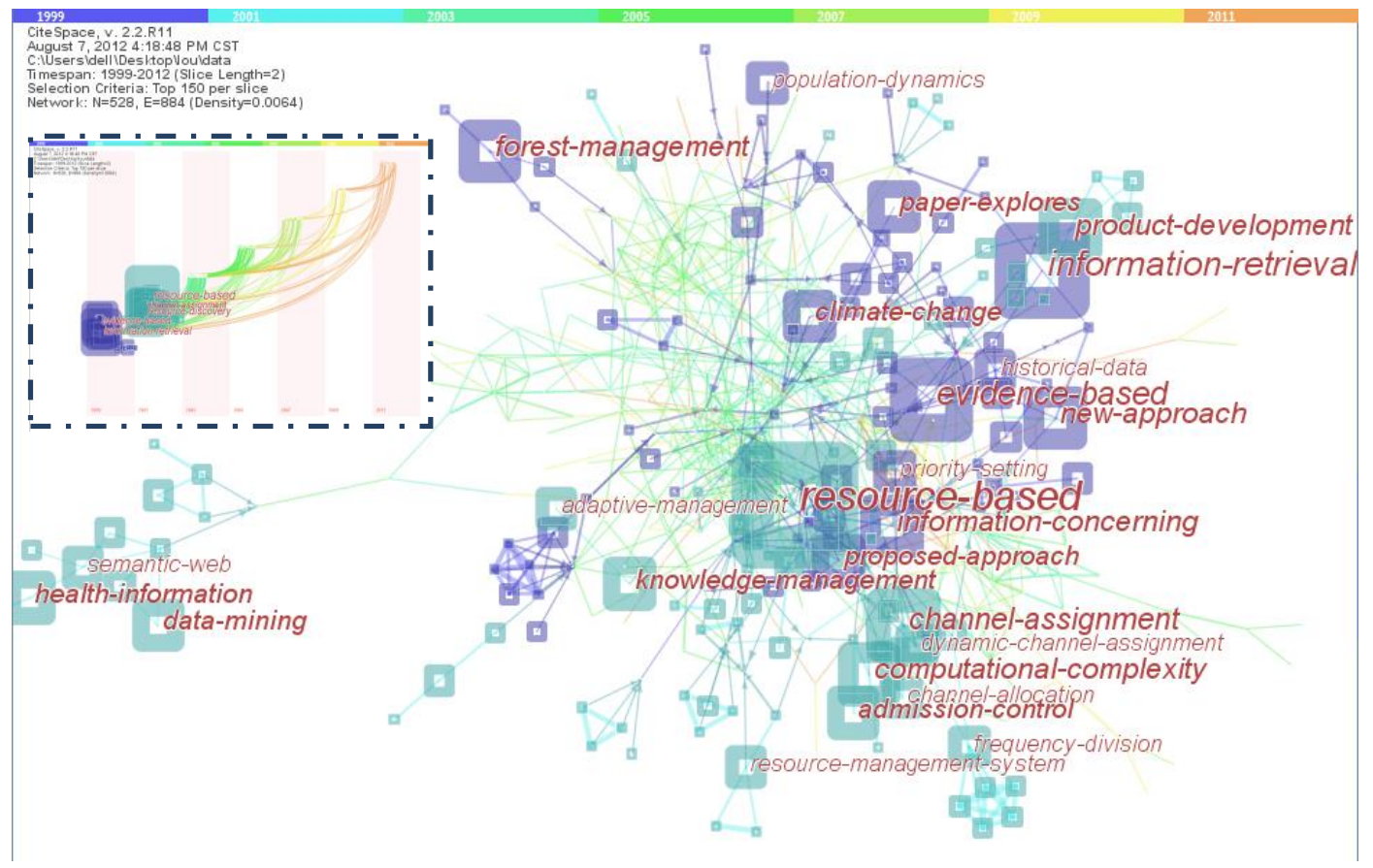

\section{Figure 3. Knowledge Map of Front Trends of Information Resource Allocation Research}

\subsection{Front Trends}

Front trends of information resource allocation research are determined by burst term detective algorithm of Citespace. The algorithm computes terms with high rate of change frequency in order to confirm trends. We get 1,007 burst terms, cluster map (Figure 3) and timeline map (top left corner of Figure 3) of frontiers. Square nodes in the map represent high rate of change frequency words, which are burst terms. The color of nodes corresponds with the color of time line. It is clear that most burst terms are purple, blue and green. Thus, burst terms mostly concentrate in the year between 1999 and 2003. Superficially, front trends do not appear in the later years. However, if we look at timeline map of frontiers, we can find out that burst terms in 1999 are still cited in 2011. So burst terms, such as resource-based and information-concerning, show front trends of information resource allocation research.

The size of nodes in Figure 3 represents the rate of change of burst terms. Table 1 shows statistics of burst terms. Burst terms, whose burst rate are bigger than 5, are resource-based, information-retrieval and evidence-based. They imply that on the one hand, front trends of information resource allocation research focus on empirical results. Most burst terms whose burst rate range between 3.1 and 5, focus on channel-assignment, new-approach, and computational-complexity (See also Table 3). Those terms are closely related to telecommunication, so computer network and telecommunication are front trends on the other hand. Additionally, some burst terms are related to natural resource and ecological allocation, such as forest-management, climate-change and health-information, which indicates that issues about bioinformatics resource and knowledge allocation are frontiers as well. 


\section{Table 3. Statistics of Burst Terms of Information Resource Allocation Research} (burst rate $\geq 3$ )

\begin{tabular}{ll||ll}
\hline burst terms & burst rate & burst terms & burst rate \\
\hline resource-based & 7.45 & product-development & 3.72 \\
information-retrieval & 5.78 & climate-change & 3.21 \\
evidence-based & 5.13 & paper-explores & 3.21 \\
channel-assignment & 4.34 & proposed-approach & 3.21 \\
new-approach & 3.85 & admission-control & 3.1 \\
forest-management & 3.85 & data-mining & 3.1 \\
information-concerning & 3.85 & health-information & 3.1 \\
computational-complexity & 3.72 & knowledge-management & 3.1 \\
\hline
\end{tabular}

\section{Conclusions}

This paper takes advantages of Citespace to conduct a comprehensive analysis of information resource allocation. CiteSpace is a powerful information visualization tool to help us uncover the structural and temporal patterns of various co-citation networks. For information resource allocation, we surpisely figure that its research firstly began with Einstein's paper. This may show how magic Citespace is.

After eighty years of development, information resource allocation research has got two periods, named foundation period and developing period. Although information resource allocation research is not going through the end period, a lot of scientists made contribution to it. Indeed, there are some leadership scholars, such as Barney, Boyd and Cover, but there is no core leader, especially in telecommunication resource research. The timeline map shows that the evolution of information resource allocation research begins with enterprise tradition resource allocation, develops to information and knowledge management, and ends up with computer network and telecommunication resource allocation from now on. The cluster map shows that hotspots and frontiers will follow resource-based theory research, empirical problems and bioinformatics resource allocation.

\section{Acknowledgements}

This paper was supported by major program of national social science foundation of China "Semantic-based Deep Integration and Visualization of Library Resources" (11\&ZD152) and the Fundamental Research Funds for the Central Universities "Semantic information retrieval based on resource ontology" (2013104010201). And I would like to thank Prof. Kou for her suggestion.

\section{References}

[1] Y. Zhao, "Study on Information Resources Collaborative Allocation in National Innovation System", Wuhan University Press, Wuhan, (2010).

[2] X. J. Zha, "Information resources allocation and sharing, Wuhan University Press, Wuhan, (2008).

[3] B. Shen, "Research on the impact that enterprise information resource allocation brings to enterprise performance", China financial \& economic publishing house, Beijing, (2009).

[4] M. Yan, "Analysis of system and incentive mechanism of information resource allocation", Southwest University Press, Nanjing, (2011).

[5] Y. F. Duan, "Web information resource allocation and regulatory mechanism: based on webometrics", Shanghai University of finance and economics press, Shanghai, (2011).

[6] Y. Zhao, "Progress in Research on Information Resources Allocation in the Innovation Environment \& Some 
Hot Topics", Information studies: theory and practice, vol. 2, no. 34, (2011).

[7] Y. S. Shen and Z. L. Sun, "A literature review of information resource allocation research", Heilongjiang History, 12, (2008).

[8] X. J. Zha and C. Cao, "Bibliometric Analysis of Information Resources Allocation Research in China over the Past 20 Years", Library and information service, vol. 10, no. 54, (2010).

[9] C. M. Chen, "CiteSpace II: Detecting and Visualizing Emerging Trends and Transient Patterns in Scientific Literature", Journal of the China Society for Scientific and Technical Information, vol. 3, (2009).

[10] A. Einstein, B. Podolsky and N. Rosen, "Can Quantum-Mechanical Description of Physical Reality Be Considered Complete?”, Physical Review, vol. 5, no. 47, (1935).

[11] R. H. Coase, "The nature of the firm", Economica-New Series, vol. 4, no. 16, (1937).

[12] E. Penrose, "The Theory of the Growth of the Firm", John Wiley and Sons, New York, (1959).

[13] S. D. Fretwell and H. L. Lucas, "On territorial behavior and other factors influencing habitat distribution in birds", Acta Biotheor, vol. 1, no. 19, (1970).

[14] B. Wernerfelt, "A resource-based view of the firm”, Strategic management journal, vol. 2, no. 5, (1984).

[15] C. E. Shannon, "A Mathematical Theory of Communication", Bell System Technical Journal, vol. 3, no. 27, (1948).

[16] G. A. Miller, "The magical number seven, plus or minus two: some limits on our capacity for processing information", Psychological Review, vol. 2, no. 63, (1956).

[17] J. Barney, "Firm Resources and Sustained Competitive Advantage", Journal of Management, vol. 17, (1991).

[18] I. Nonaka and H. Takeuchi, "The knowledge creating company: how Japanese companies create the dynamics of innovation", Oxford University Press, New York, (1995).

[19] P. L. Huang, "Enterprise Ability: The Theory Limits and the Relation Discrimination between Static Ability and Dynamic Ability", Science of Science and Management of S.\& T. 7, (2008).

[20] T. Cover and J. A. Thomas, "Elements of Information Theory", John Wiley and Sons, New York, (1991).

[21] K. P. Burnham and D. R. Anderson, "Model Selection and Multimodel Inference: A Practical InformationTheoretic Approach, Springer, New York, (2002).

[22] S. Boyd and L. Vandenberghe, "Convex Optimization", Cambridge University Press, Cambridge, (2004).

[23] D. Bertsekas, "Nonlinear programming", Athena Scientific, Nashua, (1999).

[24] S. F. Altschul, W. Gish, W. Miller, W. M. Eugene and D. J. Lipman, "Basic local alignment search tool", Journal of Molecular Biology, vol. 3, no. 215, (1990).

[25] S. F. Altschul, T. L. Madden, A. A. Schäffer, J. Zhang, Z. Zhang, W. Miller and D. J. Lipman, "Gapped BLAST and PSI-BLAST: a new generation of protein database search programs", Nucleic Acids Research, vol. 17, no. 25, (1997).

[26] M. Ashburner, C. A. Ball, J. A. Blake, D. Botstein, H. Butler, J. M. Cherry, A. P. Davis, K. Dolinski, S. S. Dwight, J. T. Eppig, M. A. Harris, D. P. Hill, L. Issel-Tarver, A. Kasarskis, S. Lewis, J. C. Matese, J. E. Richardson, M. Ringwald, G. M. Rubin and G. Sherlock, "Gene Ontology: tool for the unification of biology", Nature Genetics, vol. 1, no. 25, (2000).

[27] A. Guisan and N. E. Zimmermann, "Predictive habitat distribution models in ecology", Ecological Modeling, vol. 135, (2000).

[28] F. and C. Kesselman, "Globus: a Metacomputing Infrastructure Toolkit", International Journal of High Performance Computing Applications, vol. 2, no. 11 (1997).

[29] F. C. Kesselman and S. Tuecke, "The Anatomy of the Grid: Enabling Scalable Virtual Organizations", International Journal of High Performance Computing Applications, vol. 3, no. 15, (2001).

[30] F. P. Kelly, A. K. Maulloo and D. K. H. Tan, "Rate control for communication networks: shadow prices, proportional fairness and stability”, Journal of the Operational Research Society, vol. 3, no. 49, (1998).

[31] A. J. Goldsmith, "Capacity of fading channels with channel side information", IEEE Transactions on Information Theory, vol. 6, no. 43, (1997).

[32] D. N. C. Tse and S. V. Hanly, "Multiaccess Fading Channels-Part I: Polymatroid Structure, Optimal Resource Allocation and Throughput Capacities", IEEE Transactions on Information Theory, vol. 7, no. 44, (1998).

[33] D. N. C. Tse and S. V. Hanly, "Linear multiuser receivers: Effective interference, effective bandwidth and user capacity", IEEE Transactions on Information Theory, vol. 2, no. 45, (1999).

[34] C. Y. Wong, "Multiuser OFDM with adaptive subcarrier, bit, and power allocation", IEEE Journal on Selected Areas in Communications, vol. 10, no. 17, (1999).

[35] P. Viswanath, D. N. C. Tse and R. Laroia, "Opportunistic Beam forming Using Dumb Antennas", IEEE Transactions on Information Theory, vol. 6, no. 48, (2002).

[36] J. N. Laneman, D. N. C. Tse and G. W. Wornell, "Cooperative Diversity in Wireless Networks: Efficient Protocols and Outage Behavior”, IEEE Transactions on Information Theory, vol. 12, no. 50, (2004).

[37] S. Haykin, "Cognitive Radio: Brain-Empowered Wireless Communications", IEEE Journal on selected areas in communications, vol. 2, no. 23, (2005). 


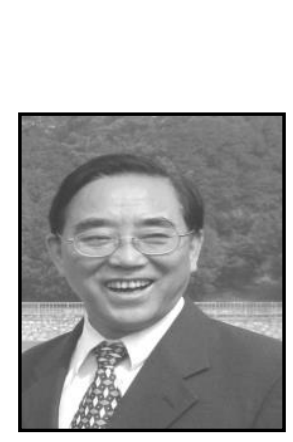

\section{Authors}

Qiu Junping, Professor and PhD supervisor of School of information management of Wuhan University, Director of Research Center of Chinese Science Evaluation. His research interests focus on informetrics, scientific evaluation, webometrics, and knowledge management.

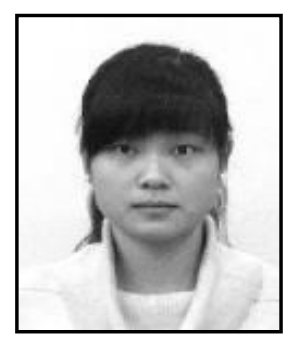

Lou Wen, PhD candidate of School of information management of Wuhan University. Her research interests focus on informetrics, digital library, semantization, and knowledge management. 
International Journal of Database Theory and Application Vol.7, No.2 (2014) 